\title{
Investigation of the Discontinuous Galerkin Method for First-Order PDE Approaches to CFD*
}

\author{
Jeffrey A. F. Hittinger ${ }^{\dagger}$ \\ Lawrence Livermore National Laboratory, Livermore, CA 94550 USA \\ Yoshifumi Suzuki ${ }^{\ddagger}$ and Bram van Leer ${ }^{\S}$ \\ University of Michigan, Ann Arbor, MI 48109 USA
}

\begin{abstract}
To simulate accurately and efficiently aerospace-type flows using solely first-order PDE models, new numerical methods may be required to overcome difficulties introduced by the model. We consider a Discontinuous Galerkin (DG) method that has been shown to possess an asymptotic preservation (AP) property for one-dimensional hyperbolic relaxation systems, in an effort to understand the source of this property and to determine if this property persists for problems in which we are interested. Comparison with traditional MUSCL schemes suggests that the source of the known AP property results from a coupling of the flux and source terms by the direct evolution of the solution slope. For a canonical model problem in a single spatial dimension, we use Von Neumann analysis to demonstrate that the limiting flux function of the DG scheme is of the Harten-Lax-Van Leer type. Further Von Neumann analysis of both a MUSCL scheme and the DG scheme show that near equilibrium, both methods have mesh size restrictions related to the resolution of physical dissipation. The DG scheme is less numerically dissipative than the HR scheme in this limit. Numerical simulations demonstrate these findings in one dimension. In one and two dimensions, our numerical results demonstrate that the convergence rate of the DG scheme eventually drops as the mesh is refined.
\end{abstract}

\section{Introduction}

In many aerospace engineering applications, advection-dominated flows are modeled adequately the compressible Navier-Stokes equations that have been the mainstay of theoretical engineering analysis. Naturally, the development of CFD methods has been influenced by this tradition. However, other PDE models may have computational advantages for approximating flows around complex geometries on modern, distributed parallel computer architectures. In particular, in comparison with second-order PDE models such as the

${ }^{*}$ This work was performed in part under the auspices of the U.S. Department of Energy by the University of California Lawrence Livermore National Laboratory under Contract W-7405-Eng-48 (UCRL-CONF-212631) and in part under the auspices of the US Department of Defense, Department of the Air Force, under Award No. F49620-03-1-0226.

${ }^{\dagger}$ Research Scientist, Center for Applied Scientific Computing, L-561, 7000 East Avenue, Member.

${ }^{\ddagger}$ Research Assistant, Department of Aerospace Engineering, 2001 François-Xavier Bagnoud Building, Student Member.

$\S$ Professor, Department of Aerospace Engineering, 3025 François-Xavier Bagnoud Building, Fellow.

Copyright (c) 2005 by the American Institute of Aeronautics and Astronautics, Inc. The U.S. Government has a royalty-free license to exercise all rights under the copyright claimed herein for Governmental purposes. All other rights are reserved by the copyright owner. 
Navier-Stokes equations, numerical methods for first-order PDE models have potentially advantageous characteristics. For example, the latter require smaller discrete stencils, reduce communications in parallel processing; can replace global stiffness from diffusion terms with local stiffness from source terms; and yield the best accuracy on non-smooth, adaptively refined grids. ${ }^{1}$

In place of second-order systems, we consider larger first-order systems of the hyperbolic-relaxation form $^{2,3}$

$$
\partial_{t} \mathbf{u}+\nabla \cdot \mathbf{f}(\mathbf{u})=\frac{1}{\tau} \mathbf{s}(\mathbf{u})
$$

where $\mathbf{u}$ is the vector of $m$ state quantities, $\mathbf{f}$ is the vector of flux vectors, $\mathbf{s}(\mathbf{u})$ is a relaxation source that operates on the time scale $\tau>0$. This is the form of moment closures of the Boltzmann equation, where the source describes departures from local thermal dynamic equilibrium. Let $T$ be the advective time scale. Near equilibrium $(\epsilon \equiv \tau / T \ll 1)$, the system formally reduces to a system of $n<m$ second-order equations

$$
\partial_{t} \mathbf{U}+\nabla \cdot \mathbf{F}(\mathbf{U})=\nabla \cdot(\mathbf{D} \nabla \mathbf{U}),
$$

where $\mathbf{D}$ is a tensor of $(n \times n)$ tensors of diffusion coefficients, with eigenvalues proportional to $\tau$. The Navier-Stokes equations have the form of $(2)$, and $(T / \tau)$ is proportional to the Reynolds number.

Numerically solving (1) can be difficult if one attempts to acquire the near-equilibrium limit using time and space meshes much coarser than the scales of the source. Consider a typical engineering calculation with characteristic length scale $L$. For a characteristic speed $a$, the residence time is $T=L / a$, and the effective relaxation length scale is $a \tau$. Clearly, when $T \gg \tau, L \gg a \tau$. Solving at scales $T$ and $L$ means that the relaxation processes are not resolved, but one wishes to obtain the asymptotic behavior of (2). As shown in, ${ }^{14,18}$ the equilibrium fluxes $\mathbf{F}$ are the result of tight coupling between the original fluxes $\mathbf{f}$ and the relaxations sources $\mathbf{s}$, so splitting techniques often fail in the sense that the order of accuracy drops. ${ }^{4-8}$

There is a large body of literature on the numerical integration of systems of the type (1), and there are many ideas and opinions as to what properties are desirable in numerical algorithms for these types of systems. One encounters many terms such as uniformly accurate, under-resolved, and asymptotic preserving, and the way in which terms are used and asymptotic limits considered are not always the same.

The general idea is that the scheme based on the full system (1) should possess, in some sense, an accurate asymptotic discretization of the continuous asymptotic limit (2). There have been many investigations into such schemes including semi-discrete approaches, ${ }^{4-6,9,10}$ characteristic approaches, ${ }^{7,11}$ centrally differenced finite-volume schemes, ${ }^{12,13}$ and modified coupled-space-time Godunov approaches. ${ }^{8,14}$ Success has not always been achieved, even within the authors' own criteria for success.

Avoiding the jargon, what we have in mind here is that we wish to discretize (1) in a formally secondorder way so that when $\epsilon$ is small, the discretization reduces to a second-order discretization of the advectivediffusive limit (2). We would prefer stable, explicit time steps that are constrained by an advective CFL

$$
2 \text { of } 26
$$


constraint, that is, $\Delta t \propto \Delta x$, where $\Delta x$ is the mesh size. We also would prefer that the mesh size be constrained only by the lengths scales of interest $L$; since we presumably are uninterested in the details of the relaxation processes, this means that we would not be required to take $\Delta x$ small enough to resolve the relaxation length $a \tau$.

Recently, Lowrie and Morel $^{15}$ analyzed and compared three semi-discrete discretizations of a simple linear model of (1). Of these, the second-order Discontinuous Galerkin (DG) discretization method was demonstrated to preserve asymptotically a diffusive limit like (2) in a single spatial dimension when the limiting advective flux was small. In fact, in this limit, the DG scheme converges with second-order accuracy for all $\Delta x / L \ll 1$, even when the cell Peclet number $\Delta x / a \tau$ is large.

However, the application of their result to our problem must be done with care because of their choice of scaling and limiting procedure, which seems more appropriate for low-speed flows. In their case, the near-equilibrium advective time scale is similar to or longer than the diffusive time scale, and so the diffusive time is the physically interesting reference time. In our case, we anticipate a near-equilibrium advective time scale much shorter than the diffusive time scale, and so we are considering an intermediate regime longer than the relaxation time but shorter than the diffusive time. This ordering of three time scales was identified in the multiple scales analysis of the model problem that we will consider. ${ }^{14,18}$

Lowrie and Morel adopted two very sensible views. First, if one is interested in the diffusive length scales, then one must use mesh sizes suitable to resolve these scales. Second, one should not expect to resolve these scales better than with a direct discretization of the asymptotic diffusion equation. For our system, these ideas relate explicitly the mesh size $\Delta x$ to some (fractional) power of the relaxation time $\tau$, since the diffusion rate scales with $\tau$. A desirable property of a discretization is that this power is less than one, so that the relaxation scale does not need to be fully resolved.

An earlier semi-discrete investigation of Godunov schemes was conducted by Jin and Levermore ${ }^{6}$ in the scaling that we will choose to use. Here, the chief constraint was that numerical dissipation be smaller than physical dissipation, which provided relationships between the mesh size $\Delta x$ and the relaxation time $\tau$. They devised modified schemes that eliminated these constraints by ensuring that the leading-order numerical dissipation terms became higher order in the asymptotic expansion. The schemes then, formally, had second-order convergence for all $\Delta x / L \ll 1$, independent of the size of $\tau / T$.

We must draw a distinction between two mesh-size constraints. Consider a direct discretization of the asymptotic limit (2). One constraint arises naturally from the discretization of the diffusion term, and that constraint requires that the wavenumbers of interest in the solution be well-resolved by the mesh size. The wavenumber content will increase in boundary layers, and the width of these regions will be related to the diffusion rate and thus $\tau$. A second constraint arises from the effective discretization of the advective 
term, which introduces additional numerical dissipation. This second constraint requires that the advective numerical dissipation be less than the physical dissipation, assuming one is interested in physical dissipation effects. This represents an additional mesh-size constraint unrelated to the discretization of the diffusion operator.

We expect the latter, advection-error based, constraint to be unavoidable for discretizations of the firstorder system (1), since it is unavoidable for discretizations of the asymptotic diffusion equation (2). Surprisingly, the work of Jin and Levermore ${ }^{6}$ suggests that schemes can be designed to overcome this constraint; in this case the mesh size is only limited by the former, resolution, constraint. We will not directly investigate the approach of Jin and Levermore here, as we will limit our consideration to the asymptotic behavior of unmodified spatial discretizations of (1).

The results of Lowrie and Morel interested us in the potential use of the DG method for discretizing firstorder systems for compressible, viscous flow. We have waded cautiously into a consideration of DG keeping in mind the aforementioned viewpoints. We note that DG has a close relationship to high-resolution Godunov (HR) methods, and consider both schemes in our analysis as a bridge between previous work. ${ }^{6,15}$ In our scaling, we find that the DG scheme converges to the advection-diffusion asymptotic limit with second-order accuracy only for mesh sizes suitable to ensure that the physical dissipation is larger than the numerical dissipation. It does behave better than the HR scheme.

In the next section, we present three model systems that will be used in the successive analysis. We consider only semi-discrete schemes and begin with a presentation of the DG and HR methods. Recasting the DG discretization in terms of the HR scheme identifies the important difference between these two methods, and this difference must be responsible for any difference between their asymptotic properties. Von Neumann analysis of the DG scheme for a linear model system identifies that an upwind DG flux function is asymptotically a Harten-Lax-Van Leer (HLL) type flux function. Continuing this harmonic analysis to higher-order terms, we identify the numerical error terms in the HR and the DG methods that compete with the physical dissipation. We present the results of a similar analysis in 2D for the DG scheme and identify a higher-order term in $\epsilon=a \tau / L$ that could dominate the convergence rate as the mesh is refined while holding $\epsilon$ fixed. We attempt to verify these results numerically in both one and two dimensions and then interpret our results in the context of previous work.

\section{Model Systems}

We consider only linear systems of the form (1) in one and two dimensions. We take the spatial and temporal variables to be dimensionless relative to the advective length and time scales. The simplest model demonstrating wave propagation in the near-equilibrium limit is a generalization of the hyperbolic heat

4 of 26

American Institute of Aeronautics and Astronautics Paper 2005-4989 
equation: 6,14

$$
\begin{aligned}
& \partial_{t} u+\partial_{x} v=0, \\
& \partial_{t} v+\partial_{x} u=-\frac{1}{\epsilon}(v-r u) .
\end{aligned}
$$

Here, $u$ is the conserved variable, $v$ is the flux of $u$, and $\epsilon>0$ is a dimensionless relaxation rate. This system has "frozen" wave speeds \pm 1 when relaxation is weak $(\epsilon \gg 1)$, and when the relaxation dominates $(\epsilon \ll 1)$,

$$
\partial_{t} u+r \partial_{x} u \approx \epsilon\left(1-r^{2}\right) \partial_{x x} u
$$

with an "equilibrium" wave speed of $r$. For stability, $|r| \leq 1$.

We note that we have written this equation in a form that leads to an advection-dominated advectiondiffusion asymptotic limit. This is consistent with a focus on compressible, viscous flow. Other choices of scalings, such as diffusive scalings, ${ }^{15,16}$ can lead to more strongly parabolic limits. Indeed, for $r=O(\epsilon)$, the scaling of Lowrie and Morel ${ }^{15}$ is in effect a long-time, small advective flux limit.

The system (3) can be generalized to break symmetry in the frozen limit:

$$
\begin{aligned}
\partial_{t} u+\partial_{x} v & =0 \\
\partial_{t} v+c \partial_{x} u+(1-c) \partial_{x} v & =-\frac{1}{\epsilon}(v-r u) .
\end{aligned}
$$

The frozen wave speeds are thus $-c$ and 1 , and the near-equilibrium form is

$$
\partial_{t} u+r \partial_{x} u \approx \epsilon(1-r)(c+r) \partial_{x x} u
$$

Note the modification to the diffusion rate. For stability, $-c \leq r \leq 1$.

In two dimensions, we consider the simple system

$$
\begin{aligned}
\partial_{t} u+\partial_{x} v+\partial_{y} w & =0 \\
\partial_{t} v+\partial_{x} u & =-\frac{1}{\epsilon}(v-r u), \\
\partial_{t} w+\partial_{y} u & =-\frac{1}{\epsilon}(w-s u),
\end{aligned}
$$

where $v$ and $w$ are the fluxes in the $x$ and $y$ directions, respectively. The near-equilibrium limit is formally

$$
\partial_{t} u+r \partial_{x} u+s \partial_{y} u \approx \epsilon\left[\left(1-r^{2}\right) \partial_{x x} u-2 r s \partial_{x y} u+\left(1-s^{2}\right) \partial_{y y}\right]
$$

with equilibrium wave speeds $r$ and $s$ in the $x$ and $y$ directions, respectively. For a harmonic factor with wave vector $\mathbf{k}=\left(k_{x}, k_{y}\right)$, a stability criteria in the near-equilibrium limit is found by insisting that the second-order derivative terms are dissipative; mathematically, this is $\left|r k_{x}+s k_{y}\right| \leq|\mathbf{k}|$. It has been shown that the near-equilibrium limit is formed by coupling between the flux and relaxation operators, ${ }^{14,18}$ and this is clear by inspection of the wave speeds and diffusion coefficients in (4), (6), and (8).

$$
5 \text { of } 26
$$




\section{Comparison of Numerical Methods}

For now, we consider the one-dimensional system

$$
\partial_{t} \mathbf{u}+\partial_{x} \mathbf{f}(\mathbf{u})=\frac{1}{\epsilon} \mathbf{s}(\mathbf{u}),
$$

where we have taken the independent variables to be normalized with the advection scales $L$ and $T(\epsilon=$ $a \tau / L)$. Within each cell $j$, the second-order DG method uses a linear basis

$$
\mathbf{u}(x)=(1-\xi) \mathbf{u}_{1}+\xi \mathbf{u}_{2}, \quad \xi=\left(x-x_{j-1 / 2}\right) / h,
$$

such that $\mathbf{u}\left(x_{j-1 / 2}\right)=\mathbf{u}_{1}$ and $\mathbf{u}\left(x_{j+1 / 2}\right)=\mathbf{u}_{2}$ are the solution values in cell $j$ at the extreme left and right edges, respectively. For linear flux $(\mathbf{f}(\mathbf{u})=\mathbf{A u})$ and source functions $(\mathbf{s}(\mathbf{u})=\mathbf{Q u})$, the semi-discrete DG scheme is then

$$
\begin{gathered}
\partial_{t} \mathbf{u}_{1}+\frac{1}{h}\left[-4 \mathbf{f}_{j-1 / 2}^{\star}-2 \mathbf{f}_{j+1 / 2}^{\star}+3 \mathbf{f}\left(\mathbf{u}_{1}\right)+3 \mathbf{f}\left(\mathbf{u}_{2}\right)\right]=\frac{1}{\epsilon} \mathbf{s}\left(\mathbf{u}_{1}\right), \\
\partial_{t} \mathbf{u}_{2}+\frac{1}{h}\left[4 \mathbf{f}_{j+1 / 2}^{\star}+2 \mathbf{f}_{j-1 / 2}^{\star}-3 \mathbf{f}\left(\mathbf{u}_{1}\right)-3 \mathbf{f}\left(\mathbf{u}_{2}\right)\right]=\frac{1}{\epsilon} \mathbf{s}\left(\mathbf{u}_{2}\right),
\end{gathered}
$$

where $\mathbf{f}_{j+1 / 2}^{\star}$ denotes the approximate flux at interface $j+1 / 2$. We will take this to be the upwind flux

$$
\mathbf{f}_{j+1 / 2}^{\star}\left(\mathbf{u}_{2, j}, \mathbf{u}_{1, j+1}\right)=\mathbf{A}^{+} \mathbf{u}_{2, j}+\mathbf{A}^{-} \mathbf{u}_{1, j+1},
$$

where, if $\boldsymbol{\Lambda}$ is the diagonal matrix of eigenvalues of $\mathbf{A}, \mathbf{A}^{ \pm}=\mathbf{R} \boldsymbol{\Lambda}^{ \pm} \mathbf{L}$.

To relate (11) to the HR method, we define

$$
\overline{\mathbf{u}}_{j}=\frac{1}{2}\left[\mathbf{u}_{1}+\mathbf{u}_{2}\right] \quad \text { and } \quad \Delta \mathbf{u}_{j}=\left[\mathbf{u}_{2}-\mathbf{u}_{1}\right]
$$

such that $\mathbf{u}(x)=\overline{\mathbf{u}}_{j}+(\xi-1 / 2) \Delta \mathbf{u}_{j}$. The DG scheme (11) is then

$$
\begin{gathered}
\partial_{t} \overline{\mathbf{u}}_{j}+\frac{1}{h}\left[\mathbf{f}_{j+1 / 2}^{\star}-\mathbf{f}_{j-1 / 2}^{\star}\right]=\frac{1}{\epsilon} \mathbf{s}\left(\overline{\mathbf{u}}_{j}\right), \\
\partial_{t} \Delta \mathbf{u}_{j}+\frac{6}{h}\left[\mathbf{f}_{j+1 / 2}^{\star}+\mathbf{f}_{j-1 / 2}^{\star}-2 \mathbf{f}\left(\overline{\mathbf{u}}_{j}\right)\right]=\frac{1}{\epsilon} \mathbf{s}\left(\Delta \mathbf{u}_{j}\right),
\end{gathered}
$$

where the upwind flux function becomes

$$
\mathbf{f}_{j+1 / 2}^{\star}\left(\mathbf{u}_{2, j}, \mathbf{u}_{1, j+1}\right) \equiv \mathbf{f}_{j+1 / 2}^{\star}\left(\overline{\mathbf{u}}_{j}+\frac{1}{2} \Delta \mathbf{u}_{j}, \overline{\mathbf{u}}_{j+1}-\frac{1}{2} \Delta \mathbf{u}_{j+1}\right) .
$$

The first update equation (14a) with (15) is precisely the HR method (modulo limiting) where $\Delta \mathbf{u}_{j} / h$ is the slope in cell $j$. For the HR method, the differences $\Delta \mathbf{u}_{j}$ are approximated at each step by differencing with neighboring cell-averaged values $\overline{\mathbf{u}}_{j}$, whereas in the DG method, the slopes evolve directly as additional variables. 
It is these slopes, whether computed or evolved, that are responsible for providing second-order spatial accuracy in the flux function evaluation. It is also these slopes that provide the difference between the two schemes. For length scales much larger than the relaxation length scale $a \tau$, the flux discretization must approximate the coupling between the two hyperbolic and relaxation operators. For an HR method, the flux function is based solely on the original hyperbolic operator and the slopes purely on the initial data. In contrast, the DG method simultaneously updates the solution average and slopes under the influence of the source.

In two dimensions, we write the linear system as

$$
\partial_{t} \mathbf{u}+\partial_{x} \mathbf{f}(\mathbf{u})+\partial_{y} \mathbf{g}(\mathbf{u})=\frac{1}{\epsilon} \mathbf{s}(\mathbf{u})
$$

with $\mathbf{g}(\mathbf{u})=\mathbf{B u}$, and we take the DG approximate to be

$$
\mathbf{u}(x, y)=\overline{\mathbf{u}}+(\xi-1 / 2) \Delta_{x} \mathbf{u}+(\eta-1 / 2) \Delta_{y} \mathbf{u},
$$

where $\xi$ is as before and $\eta=\left(y-y_{j-1 / 2}\right) / h_{y}$. The semi-discrete scheme is

$$
\begin{gathered}
\partial_{t} \overline{\mathbf{u}}_{i j}=-\frac{1}{h_{x}}\left[\mathbf{f}_{i+1 / 2, j}^{\star}-\mathbf{f}_{i-1 / 2, j}^{\star}\right]-\frac{1}{h_{y}}\left[\mathbf{g}_{i, j+1 / 2}^{\star}-\mathbf{g}_{i, j-1 / 2}^{\star}\right]+\frac{1}{\epsilon} \mathbf{s}\left(\overline{\mathbf{u}}_{i j}\right), \\
\partial_{t}\left(\Delta_{x} \mathbf{u}_{i j}\right)=-\frac{6}{h_{x}}\left[\mathbf{f}_{i+1 / 2, j}^{\star}+\mathbf{f}_{i-1 / 2, j}^{\star}-2 \mathbf{f}\left(\overline{\mathbf{u}}_{i j}\right)\right]+\frac{1}{\epsilon} \mathbf{s}\left(\Delta_{x} \mathbf{u}_{i j}\right), \\
\partial_{t}\left(\Delta_{y} \mathbf{u}_{i j}\right)=-\frac{6}{h_{y}}\left[\mathbf{g}_{i, j+1 / 2}^{\star}+\mathbf{g}_{i, j-1 / 2}^{\star}-2 \mathbf{g}\left(\overline{\mathbf{u}}_{i j}\right)\right]+\frac{1}{\epsilon} \mathbf{s}\left(\Delta_{y} \mathbf{u}_{i j}\right),
\end{gathered}
$$

where

$$
\begin{aligned}
\mathbf{f}_{i+1 / 2, j}^{\star} & =\mathbf{f}^{\star}\left(\overline{\mathbf{u}}_{i, j}+\frac{1}{2} \Delta_{x} \mathbf{u}_{i, j}, \overline{\mathbf{u}}_{i+1, j}-\frac{1}{2} \Delta_{x} \mathbf{u}_{i+1, j}\right), \\
\mathbf{g}_{i, j+1 / 2}^{\star} & =\mathbf{g}^{\star}\left(\overline{\mathbf{u}}_{i, j}+\frac{1}{2} \Delta_{y} \mathbf{u}_{i, j}, \overline{\mathbf{u}}_{i, j+1}-\frac{1}{2} \Delta_{y} \mathbf{u}_{i, j+1}\right),
\end{aligned}
$$

are one-dimensional upwind flux functions (12).

\section{Limiting Flux Function}

It is interesting to conduct a Von Neumann analysis of the one-dimensional DG method (14) for the asymmetric system (5) as $\epsilon \rightarrow 0$. The DG scheme can be written as

$$
\left(\mathbf{I} \partial_{t}+\mathbf{M}_{D G}-\mathbf{Z}_{D G}\right) \mathbf{u}_{j}=0
$$

where $\mathbf{u}_{j}=\left(\overline{\mathbf{u}}_{j}, \Delta \mathbf{u}_{j}\right)^{T} ; \mathbf{I}, \mathbf{M}_{D G}$, and $\mathbf{Z}_{D G}$ are matrices in $\mathbb{R}^{4 \times 4} ; \mathbf{I}$ is the identity matrix; and

$$
\mathbf{Z}_{D G}=\frac{1}{\epsilon}\left(\begin{array}{cc}
\mathbf{Q} & 0 \\
0 & \mathbf{Q}
\end{array}\right)
$$

$$
7 \text { of } 26
$$


If we define the forward $\delta^{+}$and backward $\delta^{-}$differencing operators and the forward $\sigma^{+}$and backward $\sigma^{-}$ sum operators such that $\delta^{+} \mathbf{u}_{j}=\mathbf{u}_{j+1}-\mathbf{u}_{j}, \delta^{-} \mathbf{u}_{j}=\mathbf{u}_{j}-\mathbf{u}_{j-1}, \sigma^{+} \mathbf{u}_{j}=\mathbf{u}_{j+1}+\mathbf{u}_{j}$, and $\sigma^{-} \mathbf{u}_{j}=\mathbf{u}_{j}+\mathbf{u}_{j-1}$, the matrix $\mathbf{M}_{D G}$ may be written as

$$
\mathbf{M}_{D G}=\frac{1}{(1+c) h}\left(\begin{array}{cccc}
c\left(\delta^{-}-\delta^{+}\right) & \delta^{-}+c \delta^{+} & c\left(\delta^{+}+\delta^{-}\right) / 2 & \left(\delta^{-}-c \delta^{+}\right) / 2 \\
c\left(\delta^{-}+c \delta^{+}\right) & \delta^{-}-c^{2} \delta^{+} & c\left(\delta^{-}-c \delta^{+}\right) / 2 & \left(\delta^{-}+c^{2} \delta^{+}\right) / 2 \\
-6 c\left(\delta^{+}+\delta^{-}\right) & 6\left(c \delta^{+}-\delta^{-}\right) & 3 c\left(\sigma^{+}+\sigma^{-}\right) & 3\left(\sigma^{-}-c \sigma^{+}\right) \\
6 c\left(c \delta^{+}-\delta^{-}\right) & -6\left(c^{2} \delta^{+}+\delta^{-}\right) & 3 c\left(\sigma^{-}-c \sigma^{+}\right) & 3\left(\sigma^{-}+c^{2} \sigma^{+}\right)
\end{array}\right)
$$

We compute the characteristic equation

$$
\operatorname{det}\left(-\lambda \mathbf{I}+\mathbf{M}_{D G}-\mathbf{Z}_{D G}\right)=0
$$

and take the equilibrium $\epsilon=0$ to obtain a quadratic equation in $\lambda$. This is

$$
\begin{aligned}
(h \lambda)^{2}-\left[\left(\frac{2 c+r(1-c)}{1+c}\right)\left(6+\delta^{+}-\delta^{-}\right)-r\left(\delta^{+}+\delta^{-}\right)\right] h \lambda & \\
& +3 r\left[r\left(\delta^{-}-\delta^{+}\right)+\left(\frac{2 c+r(1-c)}{1+c}\right)\left(\delta^{+}+\delta^{-}\right)\right]=0 .
\end{aligned}
$$

Let $\mathbf{u}_{j}=\hat{\mathbf{u}}_{0} \exp (i 2 \pi j / N)=\hat{\mathbf{u}}_{0} \exp (i \beta j)$, then (24) takes the form

$$
\begin{aligned}
(h \lambda)^{2}-2\left[\left(\frac{2 c+r(1-c)}{1+c}\right)(2+\cos \beta)-i r \sin \beta\right] & h \lambda \\
& +6 r\left[r(1-\cos \beta)+\left(\frac{2 c+r(1-c)}{1+c}\right) i \sin \beta\right]=0 .
\end{aligned}
$$

Consider the DG discretization of the equilibrium advection equation $\partial_{t} u+r \partial_{x} u=0$ obtained by taking $\epsilon=0$ in (4):

$$
\begin{gathered}
\partial_{t} u_{1, j}+\frac{1}{h}\left[-4 f_{j-1 / 2}^{q}-2 f_{j+1 / 2}^{q}+3 r\left(u_{1, j}+u_{2, j}\right)\right]=0 \\
\partial_{t} u_{2, j}+\frac{1}{h}\left[4 f_{j+1 / 2}^{q}+2 f_{j-1 / 2}^{q}-3 r\left(u_{1, j}+u_{2, j}\right)\right]=0 .
\end{gathered}
$$

We substitute for $f^{q}$ a $q$-scheme, ${ }^{19}$ that is,

$$
\begin{aligned}
f_{j+1 / 2}^{q}\left(u_{2, j}, u_{1, j+1}\right) & =\frac{r}{2}\left(u_{2, j}+u_{1, j+1}\right)-\frac{q}{2}\left(u_{1, j+1}-u_{2, j}\right), \\
& =\left(\frac{r}{2} \sigma^{+}-\frac{q}{2} \delta^{+}\right) \bar{u}_{j}-\left(\frac{r}{4} \delta^{+}-\frac{q}{4} \sigma^{+}\right) \Delta u_{j} .
\end{aligned}
$$

Similar to (20), we solve for the characteristic equation of the discrete system and insert the harmonic factor to find

$$
(h \lambda)^{2}-2[q(2+\cos \beta)-i r \sin \beta] h \lambda+6 r[r(1-\cos \beta)+i q \sin \beta]=0,
$$


which is precisely the form of $(25)$ with $q=(2 c+r(1-c)) /(1+c)$. This $q$ corresponds to the first HartenLax-Van Leer (HLL1) flux function ${ }^{20}$ based on the frozen wave speeds $a_{L}=|-c|$ and $a_{R}=1$, where

$$
f_{H L L}\left(u_{L}, u_{R}\right)=\frac{a_{L}}{a_{R}+a_{L}} f\left(u_{R}\right)+\frac{a_{R}}{a_{R}+a_{L}} f\left(u_{L}\right)-\frac{a_{R} a_{L}}{a_{R}+a_{L}}\left(u_{R}-u_{L}\right),
$$

with $f_{L \mid R}=r u_{L \mid R}$. Thus the equilibrium (and near-equilibrium) asymptotic flux function for the DG scheme applied to the simple one-dimensional problem is just the HLL1 flux function based on the frozen wave speeds.

This is not completely surprising, as a stable relaxation system has a subcharacteristic condition, that is, each equilibrium wave speed must be bounded by two frozen wave speeds. ${ }^{2,3}$ Since the upwind flux function in the DG discretization only knows about the frozen wave speeds, it continues to use them to approximate the included equilibrium wave speed. Of course, this means that the equilibrium method is not strictly upwind.

\section{Physical versus Numerical Dissipation}

In one dimension, we can apply Von Neumann analysis to both the HR and DG discretizations of (3). By this we can show, to a given order in $\epsilon$, if the scheme captures the advection-dominated advection-diffusion limit (4) with second-order accuracy in $h$. Similar analyses have been done using modified differential equations, ${ }^{6,15}$ though not always using the same scaling and limiting technique.

We first note that, for the advection-diffusion limit (4), the exact eigenvalue for the harmonic mode $\mathbf{u}=\hat{\mathbf{u}}_{0} \exp (-\lambda t+i k x)$ is

$$
\lambda=i r k+\epsilon\left(1-r^{2}\right) k^{2} .
$$

Note further that (30) has the form of a truncated power series in $\epsilon$. We will make use of this fact by assuming a power series form

$$
\lambda=\lambda_{0}+\epsilon \lambda_{1}+\epsilon^{2} \lambda_{2}+\ldots
$$

for $\epsilon \ll 1$ in order to obtain explicit forms for roots of polynomials of degree greater than two.

\section{A. HR Scheme}

The HR scheme applied to the GHHE equation (3) has the compact form

$$
\left(\mathbf{I} \partial_{t}+\mathbf{M}_{H R}-\mathbf{Q}\right) \overline{\mathbf{u}}_{j}=0
$$

where $\overline{\mathbf{u}}_{j}=\left(\bar{u}_{j}, \bar{v}_{j}\right)^{T}$ and $\mathbf{M}_{H R}$ is

$$
\begin{gathered}
\mathbf{M}_{H R}=\frac{1}{8 h}\left(\begin{array}{cc}
\left(\delta^{+}\right)^{2}+\left(\delta^{-}\right)^{2}-2\left(\delta^{+}-\delta^{-}\right) & 4\left(\delta^{+}+\delta^{-}\right)-\left(\delta^{+}\right)^{2}+\left(\delta^{-}\right)^{2} \\
4\left(\delta^{+}+\delta^{-}\right)-\left(\delta^{+}\right)^{2}+\left(\delta^{-}\right)^{2} & \left(\delta^{+}\right)^{2}+\left(\delta^{-}\right)^{2}-2\left(\delta^{+}-\delta^{-}\right)
\end{array}\right) . \\
\begin{array}{c}
9 \text { of } 26 \\
\text { American Institute of Aeronautics and Astronautics Paper 2005-4989 }
\end{array}
\end{gathered}
$$


We compute the quadratic characteristic equation

$$
\operatorname{det}\left(-\lambda \mathbf{I}+\mathbf{M}_{H R}-\mathbf{Q}\right)=0
$$

which has the two roots

$$
\lambda_{I, I I}^{H R}=\frac{(1-\cos \beta)^{2}}{2 h}+\frac{1}{2 \epsilon}\left[1 \mp \sqrt{1-2 i r \frac{\epsilon}{h} \gamma(\beta)+\left(\frac{\epsilon}{h}\right)^{2} \gamma^{2}(\beta)}\right]
$$

where $\gamma(\beta)=(3-\cos \beta) \sin \beta$. We expand the trigonometric factors in (35) for $\beta \ll 1$, the long wave-length limit, and then expand the square root. The results are

$$
\begin{aligned}
& \lambda_{I}^{H R}=i r k+\epsilon\left(1-r^{2}\right) k^{2}+\epsilon^{2} i 2 r\left(1-r^{2}\right) k^{3}+i \frac{r h^{2}}{12} k^{3}+\left[\frac{h^{3}}{8}+\epsilon \frac{\left(1-r^{2}\right)}{6} h^{2}\right] k^{4}+\ldots, \\
& \lambda_{I I}^{H R}=\frac{1}{\epsilon}-i r k-\epsilon\left(1-r^{2}\right) k^{2}-\epsilon^{2} i 2 r\left(1-r^{2}\right) k^{3}-i \frac{r h^{2}}{12} k^{3}+\left[\frac{h^{3}}{8}-\epsilon \frac{\left(1-r^{2}\right)}{6} h^{2}\right] k^{4}+\ldots,
\end{aligned}
$$

where we have defined the wavenumber $k=\beta / h$. The second root (36b) exhibits rapid exponential decay for $\epsilon \ll 1$, while the first root (36a) does not, so the latter is the dominant behavior in this asymptotic limit.

Comparing (36a) with (30), both dispersive and dissipative errors are present. The dispersive errors are second-order in $h$ or $\epsilon$, and since the correct limit shows no dispersion, these numerical dispersion errors cannot be confused with any physical dispersion. However, the leading dissipative error term, $h^{3} k^{4} / 8$, does not scale with $\epsilon$, and so can compete with the physical dissipation $\epsilon\left(1-r^{2}\right)$ if the relaxation scale is unresolved $(h \gg \epsilon)$. For the physical dissipation to dominate,

$$
h \ll 2\left[\left(1-r^{2}\right) \epsilon|k|^{-2}\right]^{1 / 3} \equiv h_{H R}^{*},
$$

so the HR scheme does not attain the asymptotic limit to second-order in $h$ with $h$ independent of $\epsilon$.

For the HR scheme, the result (37) is well known. It appears for the $r=0$ case in previous studies, ${ }^{6,15}$ although not necessarily in our scaling. The form (36a) for $r \neq 0$ for the HR scheme can be obtained from Eq. (3.17) in Jin and Levermore ${ }^{6}$ or Eq (31) in Lowrie and Morel. ${ }^{15}$ In the scaling of the latter work, the term that leads to (37) is actually divergent since it goes like $h^{3} / \epsilon$ (due to the time dilation of this scaling), but it still leads to the constraint (37).

It is interesting to compare (37) with a direct discretization of the asymptotic equation using the LaxFriedrichs flux function (4) and slope reconstruction. The diffusion term is discretized using a three-point, second-order central difference approximation. From Von Neumann analysis, the eigenvalue of the scheme is

$$
\lambda_{a d}^{H R}=i r k+\epsilon\left(1-r^{2}\right) k^{2}+i \frac{r h^{2}}{12} k^{3}+\left[\frac{h^{3}}{8}-\epsilon \frac{\left(1-r^{2}\right)}{12} h^{2}\right] k^{4}+\ldots,
$$

for $\epsilon \ll 1$ and $h \ll 1$. We see that this has the same fourth-order numerical dissipation term as (36a), which in this case arises directly form the advection discretization. This discretization will have the same restriction (37) on $h$ to ensure that the physical dissipation is dominant. 


\section{B. DG Scheme}

The DG scheme in compact form is given by (20); we specialize here to the case of $c=1$. Since this is a $4 \times 4$ system, the characteristic polynomial is of degree four, and it is therefore difficult to write the complete roots explicitly. Instead we perturbatively construct the eigenvalues order by order, assuming the power series form (31). The leading-order behavior is given by the quadratic equation (25). Continuing in this way out through terms of $O(\epsilon)$, and, at each order, expanding out the trigonometric terms for $\beta \ll 1$, we find

$$
\begin{aligned}
& \lambda_{I}^{D G}=i r \frac{\beta}{h}+\epsilon\left(1-r^{2}\right) \frac{\beta^{2}}{h^{2}}+\ldots, \\
& \lambda_{I I}^{D G}=\frac{1}{\epsilon}-i r \frac{\beta}{h}-\epsilon\left(1-r^{2}\right) \frac{\beta^{2}}{h^{2}}+\ldots, \\
& \lambda_{I I I}^{D G}=\frac{6}{h}-3 i r \frac{\beta}{h}-\left[h-9 \epsilon\left(1-r^{2}\right)\right] \frac{\beta^{2}}{h^{2}}+\ldots, \\
& \lambda_{I V}^{D G}=\frac{1}{\epsilon}+\frac{6}{h}+3 i r \frac{\beta}{h}-\left[h-9 \epsilon\left(1-r^{2}\right)\right] \frac{\beta^{2}}{h^{2}}+\ldots
\end{aligned}
$$

The last three roots all exhibit rapid exponential decay for $h \ll 1$ and $\epsilon \ll 1$, while the first root does not.

Since $\lambda_{I}^{D G}$ is the dominant root in the asymptotic limit, we continue expanding it out through terms of $O\left(\epsilon^{2}\right)$, and, expanding out the trigonometric factors, we find

$$
\lambda_{I}^{D G}=i r k+\epsilon\left(1-r^{2}\right) k^{2}+\epsilon^{2} 2 i r\left(1-r^{2}\right) k^{3}+\left[\frac{r^{2} h^{3}}{72}+\epsilon \frac{\left(1-r^{2}\right)}{12} h^{2}-\epsilon^{2} \frac{1-r^{2}}{2} h\right] k^{4}+\ldots,
$$

where we have made the substitution $k=\beta / h$. Again, we find a fourth-order numerical dissipation term independent of $\epsilon$ that can compete with the physical second-order dissipation. The criteria for the physical dissipation to dominate is

$$
h \ll 2\left[\frac{9\left(1-r^{2}\right)}{r^{2}} \epsilon|k|^{-2}\right]^{1 / 3} \equiv h_{D G}^{*},
$$

which is a factor of $\left(9 / r^{2}\right)^{1 / 3}$ larger than for the HR scheme. When rescaled, this is the same result as Eq. (32) in Lowrie and Morel ${ }^{15}$ that obtained from modified differential equation analysis.

We directly discretize the advection-diffusion limit (4) using the DG scheme with a Lax-Friedrichs flux function. ${ }^{a}$ The diffusion term is discretized using the recently developed ${ }^{17}$ "recovery method". The dominant eigenvalue from the Von Neumann analysis is

$$
\lambda_{a d}^{D G}=i r k+\epsilon\left(1-r^{2}\right) k^{2}+\left[\frac{r^{2} h^{3}}{72}-\epsilon \frac{5 r^{2}\left(1-r^{2}\right)}{144} h^{2}\right] k^{4}+\ldots,
$$

for $\epsilon \ll 1$ and $h \ll 1$. Again, the dominant numerical dissipation is of precisely the same form as for the GHHE discretization (40).

Finally, we note that, for $r=o(\epsilon)$ with $\epsilon \ll 1$, the DG scheme exhibits an interesting property. In this case, the fourth-order numerical dissipation term in (40) becomes higher-order in $\epsilon$, and the constraint (41)

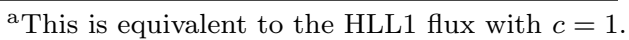


on $h$ is removed. Thus, the DG scheme should converge with second-order accuracy in $h$ with $h$ independent of $\epsilon$, in so far as even higher-order terms do not compete with the physical dissipation. This case is included in the diffusive limit considered by Lowrie and Morel, ${ }^{15}$ and our result agrees with theirs, when one accounts for the time dilation of their scaling.

\section{2D GHHE Von Neumann Analysis}

The two-dimensional DG discretization can be written in the compact form

$$
\left(\mathbf{I} \partial_{t}+\mathbf{M}_{x}+\mathbf{M}_{y}-\mathbf{Z}\right) \mathbf{u}_{j k}=0
$$

where

$$
\mathbf{u}_{j k}=\left[\overline{\mathbf{u}}_{j k}, \overline{\Delta \mathbf{u}}_{j k}\right]^{T}=\left[\bar{u}_{j k}, \bar{v}_{j k}, \bar{w}_{j k},{\overline{\Delta_{x}}}_{j k},{\overline{\Delta_{x} v}}_{j k},{\overline{\Delta_{x} w_{j k}}}_{, \bar{\Delta}_{y} u},{\overline{\Delta_{y} v}}_{j k},{\overline{\Delta_{y} w_{j k}}}\right]^{T},
$$

and matrix operators of spatial differentiation in $x$ - and $y$-direction are given by

$$
\begin{gathered}
\mathbf{M}_{x}=\frac{1}{2 \Delta x}\left(\begin{array}{ccc}
\mathbf{D}_{x}^{-} & \mathbf{D}_{x}^{+} / 2 & \mathbf{0} \\
-6 \mathbf{D}_{x}^{+} & -3\left(\mathbf{D}_{x}^{-}-4 \mathbf{I}\right) & \mathbf{0} \\
\mathbf{0} & \mathbf{0} & \mathbf{0}
\end{array}\right), \mathbf{M}_{y}=\frac{1}{2 \Delta y}\left(\begin{array}{ccc}
\mathbf{D}_{y}^{-} & \mathbf{0} & \mathbf{D}_{y}^{+} / 2 \\
\mathbf{0} & \mathbf{0} & \mathbf{0} \\
-6 \mathbf{D}_{y}^{+} & \mathbf{0} & -3\left(\mathbf{D}_{y}^{-}-4 \mathbf{I}\right)
\end{array}\right), \\
\mathbf{D}_{x}^{+}=\left(\begin{array}{ccc}
\delta_{x}^{+}+\delta_{x}^{-} & \delta_{x}^{-}-\delta_{x}^{+} & 0 \\
\delta_{x}^{-}-\delta_{x}^{+} & \delta_{x}^{+}+\delta_{x}^{-} & 0 \\
0 & 0 & 0
\end{array}\right), \mathbf{D}_{x}^{-}=\left(\begin{array}{ccc}
\delta_{x}^{-}-\delta_{x}^{+} & \delta_{x}^{+}+\delta_{x}^{-} & 0 \\
\delta_{x}^{+}+\delta_{x}^{-} & \delta_{x}^{-}-\delta_{x}^{+} & 0 \\
0 & 0 & 0
\end{array}\right), \\
\mathbf{D}_{y}^{+}=\left(\begin{array}{ccc}
\delta_{y}^{+}+\delta_{y}^{-} & 0 & \delta_{y}^{-}-\delta_{y}^{+} \\
0 & 0 & 0 \\
\delta_{y}^{-}-\delta_{y}^{+} & 0 & \delta_{y}^{+}+\delta_{y}^{-}
\end{array}\right), \mathbf{D}_{y}^{-}=\left(\begin{array}{ccc}
\delta_{y}^{-}-\delta_{y}^{+} & 0 & \delta_{y}^{+}+\delta_{y}^{-} \\
0 & 0 & 0 \\
\delta_{y}^{+}+\delta_{y}^{-} & 0 & \delta_{y}^{-}-\delta_{y}^{+}
\end{array}\right) .
\end{gathered}
$$

The source term matrix is defined to be

$$
\mathbf{Z}=\left(\begin{array}{ccc}
\mathbf{Q} & \mathbf{0} & \mathbf{0} \\
\mathbf{0} & \mathbf{Q} & \mathbf{0} \\
\mathbf{0} & \mathbf{0} & \mathbf{Q}
\end{array}\right), \mathbf{Q}=\frac{1}{\epsilon}\left(\begin{array}{ccc}
0 & 0 & 0 \\
r & -1 & 0 \\
s & 0 & -1
\end{array}\right)
$$

We restrict consideration to the $r=s=0$ case and further specialize to a uniform grid with unit aspect ratio $(h \equiv \Delta x / L=\Delta y / L)$. Let $\mathbf{u}_{j k}=\hat{\mathbf{u}}_{0} \exp (i \alpha j) \exp (i \beta k)$. We look for roots of the characteristic polynomial

$$
\operatorname{det}\left(-\lambda \mathbf{I}+\mathbf{M}_{x}+\mathbf{M}_{y}-\mathbf{Z}\right)
$$

We will skip consideration of the sub-dominant eigenvalues and further specialize our analysis to $\alpha=\beta$.

12 of 26 
As in 1D, we assume a power series form for the eigenvalue, solve for the eigenvalue order-by-order in $\epsilon$, and expand trigonometric factors for $\beta \ll 1$. We find the dominant eigenvalue

$$
\lambda=2 \epsilon k^{2}-\epsilon^{2} k^{4} h+\frac{\epsilon^{2}}{18} k^{6} h^{3}-\frac{\epsilon}{120} k^{6} h^{4}+\ldots,
$$

where $k=\beta / h$. The first term is the physical dissipation.

Consider $\epsilon \ll 1$ and fixed. Likewise, take $k$ constant. For coarse resolution, the last term typically will dominate. As the mesh size is reduced, the second term, which is first-order in $h$ will eventually dominate. The threshold grid size for this turnover is

$$
h=\left[120 \epsilon k^{-2}\right]^{1 / 3}
$$

For $\epsilon=10^{-5}$ and $k=2 \pi$, this gives $N=1 / h \approx 32$. This is similar to the term in the $1 \mathrm{D}$ DG discretization, but it appears that its effects are more pronounced.

\section{Numerical Experiments}

It is instructive to demonstrate numerically the behavior of these two schemes in the context of this analysis. Recall that there is still a temporal stiffness issue which must be overcome by using some implicit integration technique. We take as an integrator the second-order, $L$-stable predictor-corrector scheme used by Lowrie and Morel: ${ }^{15}$

$$
\begin{aligned}
\frac{\mathbf{u}^{n+1 / 2}-\mathbf{u}^{n}}{\Delta t / 2} & =\mathbf{H}\left(\mathbf{u}^{n}\right)+\frac{1}{\epsilon} \mathbf{S}\left(\mathbf{u}^{n+1 / 2}\right), \\
\frac{\left(\mathbf{u}^{n+1}+\mathbf{u}^{*}\right) / 2-\mathbf{u}^{n}}{\Delta t / 2} & =\mathbf{H}\left(\mathbf{u}^{n+1 / 2}\right)+\frac{1}{\epsilon} \mathbf{S}\left(\mathbf{u}^{*}\right), \\
\frac{\left(\mathbf{u}^{n+1}-\mathbf{u}^{*}\right)}{\Delta t} & =\mathbf{H}\left(\mathbf{u}^{n+1 / 2}\right)+\frac{1}{\epsilon} \mathbf{S}\left(\mathbf{u}^{n+1}\right),
\end{aligned}
$$

where $\mathbf{H}(\mathbf{u})$ and $\mathbf{S}(\mathbf{u})$ represent the discrete differential and source operators, respectively.

\section{A. One Dimensional Tests}

For each scheme, we consider an initial value problem on a periodic domain, and compute the error norms relative to an exact analytic solution. We consider both a non-stiff $(\epsilon \gg 1)$ and a stiff $(\epsilon \ll 1)$ case. 


\section{Problem Definition}

We consider the symmetric model problem (3). The DG method requires cell averages $\overline{\mathbf{u}}_{j}$ and slopes $\Delta \mathbf{u}_{j}$ in each cell for initial conditions. Given an initial function $\mathbf{u}(x, 0)$, the initial cell values are obtained ${ }^{21}$ from

$$
\begin{aligned}
\overline{\mathbf{u}}_{j} & =\frac{1}{h} \int_{x_{j-1 / 2}}^{x_{j+1 / 2}} \mathbf{u}(x, 0) d x \\
\Delta_{x} \mathbf{u}_{j} & =\frac{\int_{x_{j-1 / 2}}^{x_{j+1}} \mathbf{u}(x, 0)\left(\frac{x-x_{j}}{h}\right) d x}{\int_{x_{j-1 / 2}}^{x_{j+1 / 2}}\left(\frac{x-x_{j}}{h}\right)^{2} d x}=\frac{12}{(h)^{2}} \int_{x_{j-1 / 2}}^{x_{j+1 / 2}} \mathbf{u}(x, 0)\left(x-x_{j}\right) d x .
\end{aligned}
$$

We take our domain $x \in[0,1]$ to be periodic and use the harmonic initial condition

$$
\mathbf{u}(x, 0)=\mathcal{R}\left\{\mathbf{u}_{0} \exp (i k x)\right\}=\mathbf{u}_{0} \cos (k x),
$$

where $k=2 \pi$ and $\mathbf{u}_{0}=(1,1)^{T}$. A dispersion analysis ${ }^{14}$ provides the exact solution for the computation of the $L_{2}$-norm. Two equilibrium wave speeds are considered: $r=1 / 2$ and $r=0$. We use fixed CFL numbers of 0.8 for the HR method and 0.3 for the DG scheme.

\section{Convergence in the Frozen Limit $\left(\epsilon=10^{3}, t_{\mathrm{end}}=10^{2}\right)$}

When the relaxation time is large relative to the residence time, the effect of source term is negligible, and the model equations behave like pure advection equations. The CFL condition based on frozen wave speeds provides a stable time step in this limit. Figure 1 and Table 1 demonstrate second-order convergence in this limit for both the DG and HR schemes for $r=1 / 2$. The asymptotic convergence regime (in $h$ ) is not entered until $h<0.05$, which is somewhat surprising. This could be an effect from the temporal discretization, because the spatial discretizations are well behaved for $\epsilon \gg 1$. The frozen-limit results for $r=0$ are nearly identical, and so have not been included.

\section{Convergence in the Near-Equilibrium Limit $\left(\epsilon=10^{-5}, t_{\mathrm{end}}=10^{3}\right)$}

When the relaxation time is small relative to the residence time, the source term is dominant, and the asymptotic equation is an advection-dominated advection-diffusion equation. We have found that, for both schemes, when $r \neq 0$, each has a mesh size threshold restriction $h \sim \epsilon^{1 / 3}|k|^{-2 / 3}$ above which numerical dissipation dominates and below which physical dissipation dominates. When $r=0$, the DG loses this restriction.

We demonstrate this numerically. As before, the time step is based solely on the frozen wave speed CFL condition. Since the diffusion is weak, the simulations are run for many time steps until a sufficient 


\begin{tabular}{cccccc}
\hline Method & $N$ & $L_{2}(u)$ & Rate & $L_{2}(v)$ & Rate \\
\hline DG & 10 & $7.737 \mathrm{e}-01$ & - & $7.736 \mathrm{e}-01$ & - \\
& 20 & $5.816 \mathrm{e}-01$ & 0.41 & $5.817 \mathrm{e}-01$ & 0.41 \\
& 40 & $1.596 \mathrm{e}-01$ & 1.87 & $1.597 \mathrm{e}-01$ & 1.87 \\
& 80 & $4.013 \mathrm{e}-02$ & 1.99 & $4.013 \mathrm{e}-02$ & 1.99 \\
& 160 & $1.003 \mathrm{e}-2$ & 2.00 & $1.003 \mathrm{e}-02$ & 2.00 \\
\hline \multirow{2}{*}{ HR } & 10 & $6.784 \mathrm{e}-01$ & - & $6.784 \mathrm{e}-01$ & - \\
& 20 & $6.368 \mathrm{e}-01$ & 0.09 & $6.368 \mathrm{e}-01$ & 0.09 \\
& 40 & $1.994 \mathrm{e}-01$ & 1.68 & $1.994 \mathrm{e}-01$ & 1.68 \\
& 80 & $3.633 \mathrm{e}-02$ & 2.46 & $3.632 \mathrm{e}-02$ & 2.46 \\
& 160 & $7.391 \mathrm{e}-03$ & 2.30 & $7.391 \mathrm{e}-03$ & 2.30 \\
\hline
\end{tabular}

Table 1. $L_{2}$-norm and rate of convergence for $r=1 / 2$ in the frozen limit $\left(\epsilon=10^{3}\right)$ for 1D GHHE system.

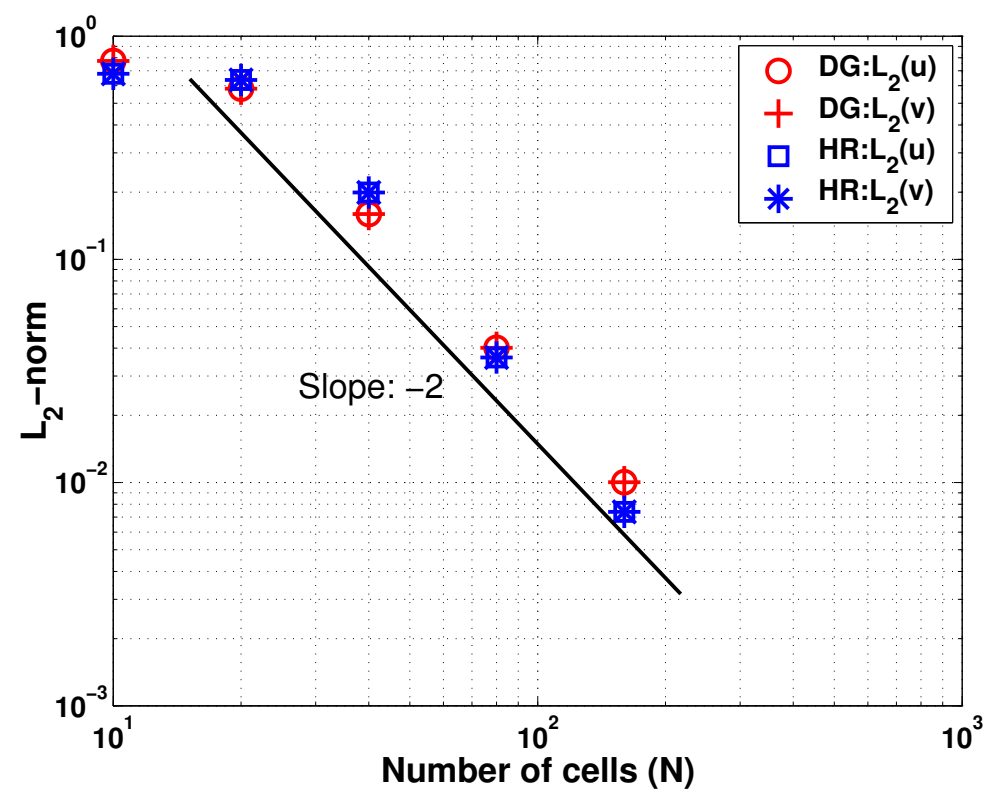

Figure 1. 1D GHHE grid convergence study in the frozen limit for $r=1 / 2$ and $\epsilon=10^{3}$. 


\begin{tabular}{ccccccc}
\hline Method & $N$ & $h / h^{*}$ & $L_{2}(u)$ & Rate & $L_{2}(v)$ & Rate \\
\hline \multirow{2}{*}{ DG } & 10 & $2.63 \mathrm{e} 0$ & $5.192 \mathrm{e}-01$ & - & $2.596 \mathrm{e}-01$ & - \\
& 20 & $1.32 \mathrm{e} 0$ & $4.600 \mathrm{e}-01$ & 0.17 & $2.300 \mathrm{e}-01$ & 0.17 \\
& 40 & $6.59 \mathrm{e}-1$ & $1.505 \mathrm{e}-01$ & 1.61 & $7.523 \mathrm{e}-01$ & 1.61 \\
& 80 & $3.29 \mathrm{e}-1$ & $3.910 \mathrm{e}-02$ & 1.94 & $1.955 \mathrm{e}-02$ & 1.94 \\
& 160 & $1.65 \mathrm{e}-1$ & $1.003 \mathrm{e}-02$ & 1.96 & $5.016 \mathrm{e}-03$ & 1.96 \\
\hline \multirow{2}{*}{ HR } & 10 & $8.70 \mathrm{e} 0$ & $5.173 \mathrm{e}-01$ & - & $2.586 \mathrm{e}-01$ & - \\
& 20 & $4.35 \mathrm{e} 0$ & $5.237 \mathrm{e}-01$ & -0.02 & $2.619 \mathrm{e}-01$ & -0.02 \\
& 40 & $2.17 \mathrm{e} 0$ & $5.104 \mathrm{e}-01$ & 0.04 & $2.552 \mathrm{e}-01$ & 0.04 \\
& 80 & $1.09 \mathrm{e} 0$ & $6.773 \mathrm{e}-01$ & -0.41 & $3.386 \mathrm{e}-01$ & -0.41 \\
& 160 & $5.44 \mathrm{e}-1$ & $2.221 \mathrm{e}-01$ & 1.61 & $1.110 \mathrm{e}-01$ & 1.61 \\
\hline
\end{tabular}

Table 2. $L_{2}$-norm and rate of convergence for $r=1 / 2$ in the near-equilibrium limit $\left(\epsilon=10^{-5}\right)$ for the 1D GHHE system.

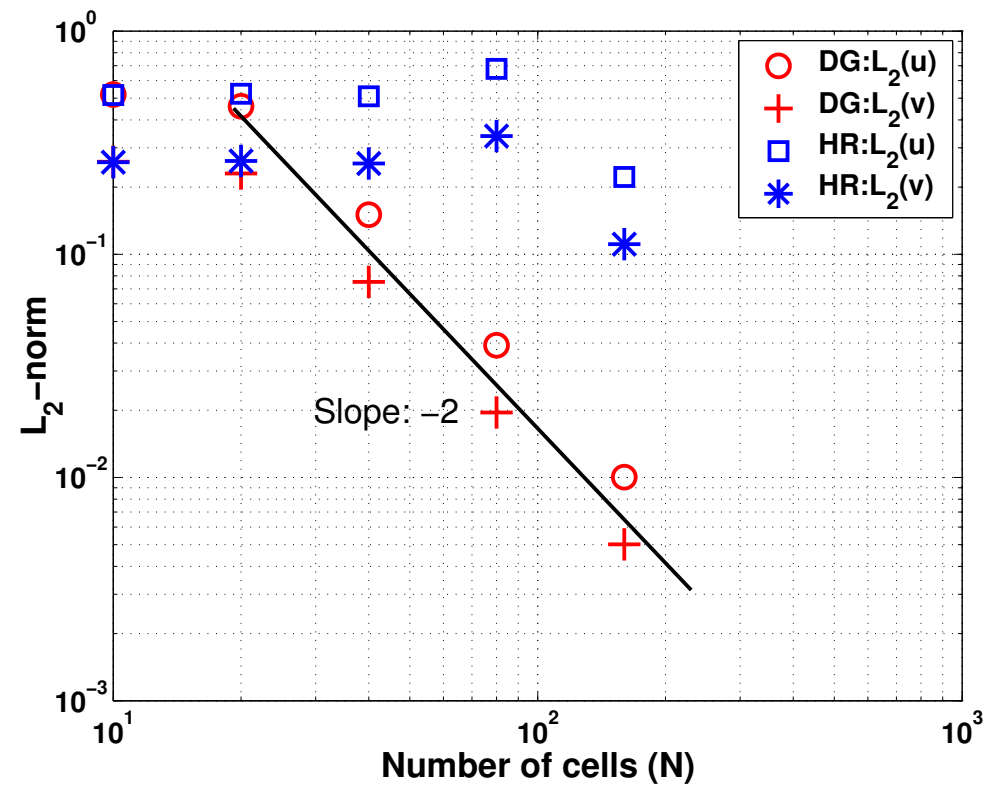

Figure 2. 1D GHHE Grid convergence study in the near-equilibrium limit for $r=1 / 2$ and $\epsilon=10^{-5}$. 
amplitude reduction can be observed.

Results for $r=1 / 2$ are shown in Table 2 and Figure 2. The HR scheme only begins to converge when $h<0.0125$, while the DG method for $h<0.05$, clearly converging with second order accuracy. The utter lack of convergence for HR can be understood by considering the values of the $L_{2}$-norms, which are roughly the $L_{2}$-norms of the exact solution. In other words, the numerical dissipation has so swamped the physical dissipation, that there is effectively no signal left.

Considering Table 2, both schemes begin to exhibit convergence precisely when the mesh size $h$ becomes smaller than the limit $h_{H R}^{*}$ or $h_{D G}^{*}$. From these results, however, it is unclear if the threshold for the DG method is an artifact of the spatial or temporal discretization. Certainly, the results agree with the threshold prediction, but the results also exhibit a behavior very similar to that in the "frozen" limit.

When the equilibrium wave speed vanishes $(r=0)$, the DG scheme should exhibit second-order convergence without the threshold. Results are provided in Table 3 and Figure 3. Indeed, the DG scheme initially converges at a second-order rate and exhibits no threshold. This suggests that the threshold for $r=1 / 2$ was in fact a demonstration of the behavior of the spatial discretization. For the smallest values of $h$ the convergence appears to slow down without any cause apparent from the eigenvalue analysis. This may be a rounding-error effect, since the error level in the flux variable $v$ has dropped to $10^{-9}$.

As before, the HR scheme exhibits a threshold and does not appear to begin to converge until $h<h_{H R}^{*}$. For $N=160$, the HR scheme appears to converge at a rate greater than two. This can be explained by the fact that, for our choice of parameters, the $h^{3} k^{4} / 8$ error term still dominates the numerical error, even if it is smaller than the physical dissipation.

\section{B. Two Dimensional Tests}

In a single dimension, the DG scheme for $r=0$ does not appear to have a threshold where numerical dissipation dominates over physical dissipation. However, problems of practical significance are multidimensional, and multiple dimensions frequently introduce additional couplings. Analysis suggests that, while the 2D DG scheme does not suffer from a problem with numerical diffusion overwhelming physical diffusion, convergence may slow to first order for small enough mesh size. To gauge whether the 2D DG scheme actually exhibits this behavior, we conduct a two-dimensional numerical experiment for $r=s=0$.

\section{Problem Definition}

We consider the two-dimensional model problem (7). In 2D, the DG method requires cell averages $\overline{\mathbf{u}}_{j k}$ and slopes $\Delta_{x} \mathbf{u}_{j k}, \Delta_{y} \mathbf{u}_{j k}$ in each cell for initial conditions. Given an initial function $\mathbf{u}(x, y, 0)$, the initial cell 


\begin{tabular}{ccccccc}
\hline \multirow{2}{*}{ Method } & $N$ & $h / h^{*}$ & $L_{2}(u)$ & Rate & $L_{2}(v)$ & Rate \\
\hline \multirow{2}{*}{ DG } & 10 & & $6.540 \mathrm{e}-03$ & - & $5.831 \mathrm{e}-07$ & - \\
& 20 & & $1.568 \mathrm{e}-03$ & 2.06 & $1.481 \mathrm{e}-07$ & 1.98 \\
& 40 & & $3.886 \mathrm{e}-04$ & 2.01 & $3.716 \mathrm{e}-08$ & 1.99 \\
& 80 & & $1.009 \mathrm{e}-04$ & 1.95 & $9.457 \mathrm{e}-09$ & 1.97 \\
& 160 & & $3.789 \mathrm{e}-05$ & 1.41 & $2.951 \mathrm{e}-09$ & 1.68 \\
\hline \multirow{2}{*}{ HR } & 10 & $7.90 \mathrm{e} 0$ & $4.687 \mathrm{e}-01$ & - & $2.945 \mathrm{e}-05$ & - \\
& 20 & $3.95 \mathrm{e} 0$ & $4.745 \mathrm{e}-01$ & -0.02 & $2.981 \mathrm{e}-05$ & -0.02 \\
& 40 & $1.98 \mathrm{e} 0$ & $4.527 \mathrm{e}-01$ & 0.07 & $2.844 \mathrm{e}-05$ & 0.07 \\
& 80 & $9.88 \mathrm{e}-1$ & $1.504 \mathrm{e}-01$ & 1.59 & $9.442 \mathrm{e}-06$ & 1.59 \\
& 160 & $4.94 \mathrm{e}-1$ & $2.211 \mathrm{e}-02$ & 2.77 & $1.385 \mathrm{e}-06$ & 2.77 \\
\hline
\end{tabular}

Table 3. $L_{2}$-norm and rate of convergence for $r=0$ in the near-equilibrium limit $\left(\epsilon=10^{-5}\right)$ for the $1 \mathrm{D}$ GHHE system.

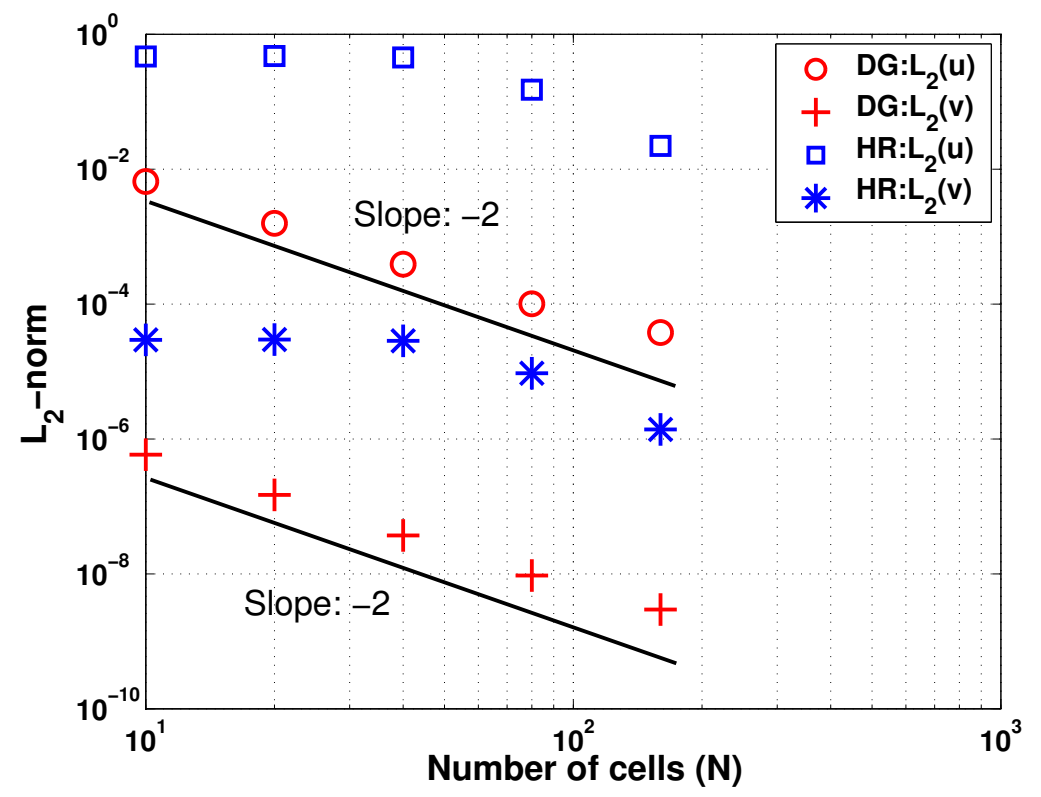

Figure 3. 1D GHHE Grid convergence study in the near-equilibrium limit for $r=0$ and $\epsilon=10^{-5}$. 
values are obtained from

$$
\begin{aligned}
\overline{\mathbf{u}}_{j k} & =\frac{1}{h_{x} h_{y}} \iint_{C_{j k}} \mathbf{u}(x, y, 0) d x d y, \\
\Delta_{x} \mathbf{u}_{j k} & =\frac{12}{h_{x}^{2} h_{y}} \iint_{C_{j k}} \mathbf{u}(x, y, 0)\left(x-x_{j}\right) d x d y, \\
\Delta_{y} \mathbf{u}_{j k} & =\frac{12}{h_{x} h_{y}^{2}} \iint_{C_{j k}} \mathbf{u}(x, y, 0)\left(y-y_{k}\right) d x d y,
\end{aligned}
$$

where the integration is over the cell $C_{j k}=\left[x_{j-1 / 2}, x_{j+1 / 2}\right] \times\left[y_{k-1 / 2}, y_{k+1 / 2}\right]$. We take our domain $x \in[0,1]$, $y \in[0,1]$ to be periodic in each direction and use the harmonic initial condition

$$
\mathbf{u}(x, y, 0)=\mathbf{u}_{0}\left[\cos \left(k_{x} x\right) \cos \left(k_{y} y\right)-\sin \left(k_{x} x\right) \sin \left(k_{y} y\right)\right]
$$

where $\mathbf{u}_{0}=[1,1,1]^{T}$ and $k_{x}=k_{y}=2 \pi$. This initial condition has variation in the diagonal direction at $45^{\circ}$ to the $x$-axis and is uniform in the other diagonal direction. The equilibrium wave speeds are set to $r=s=0$, and a fixed CFL limit of 0.3 is used. A dispersion analysis, included in Appendix A, provides the exact solution for the computation of the $L_{2}$-norm.

\section{Convergence in the Frozen Limit $\left(\epsilon=10^{3}, t_{\mathrm{end}}=10^{2}\right)$}

Figure 4 and Table 4 demonstrate the expected second-order convergence for the 2D DG method in the frozen limit, although the onset of the asymptotic convergence rate requires slightly more points per dimension than in $1 \mathrm{D}$. We note that the frozen problem propagates the initial condition along the diagonal of the domain, and this misalignment of the propagation direction and the grid produces an effective mesh size in the propagation direction larger than $h$ by a factor of $\sqrt{2}$. This factor is enough to explain the difference between the 1D and $2 \mathrm{D}$ results.

\section{Convergence in the Near-Equilibrium Limit $\left(\epsilon=10^{-5}, t_{\mathrm{end}}=10^{3}\right)$}

Results are shown in Table 5 and Figure 5 . The fluxes $v$ and $w$ appear to converge with a second-order rate in $h$, at least until $N_{x}=N_{y}=160$. The solution $u$ at first converges at a fourth-order rate, which seemingly transitions to a third-order rate, before finally stalling. The transition to a regime of no convergence occurs before the expected transition to first order that our analysis suggested. Most likely, the third-order rate is the combined effect of the fourth-order error term and whatever is stalling the convergence, in which case the initial convergence rate follows the behavior predicted in our analysis.

As in the $1 \mathrm{D}$ results, although demonstrated much more dramatically here, the convergence seems to stall when the numerical error approaches the size of the relaxation parameter $\epsilon$. An obvious conjecture is 


\begin{tabular}{cccccc}
\hline Method & $N_{x} \times N_{y}$ & $L_{2}(u)$ & Rate & $L_{2}(v)$ & Rate \\
\hline DG & $10 \times 10$ & $7.23 \mathrm{e}-01$ & - & $6.14 \mathrm{e}-01$ & - \\
& $20 \times 20$ & $1.05 \mathrm{e}-00$ & -0.54 & $9.34 \mathrm{e}-01$ & -0.60 \\
& $40 \times 40$ & $5.33 \mathrm{e}-01$ & 0.98 & $4.31 \mathrm{e}-01$ & 1.11 \\
& $80 \times 80$ & $1.59 \mathrm{e}-01$ & 1.74 & $1.03 \mathrm{e}-01$ & 2.06 \\
& $160 \times 160$ & $4.13 \mathrm{e}-02$ & 1.95 & $2.51 \mathrm{e}-02$ & 2.04 \\
\hline
\end{tabular}

Table 4. $L_{2}$-norm and rate of convergence for $r=s=0$ in the frozen limit $\left(\epsilon=10^{3}\right)$ for the 2D GHHE system.

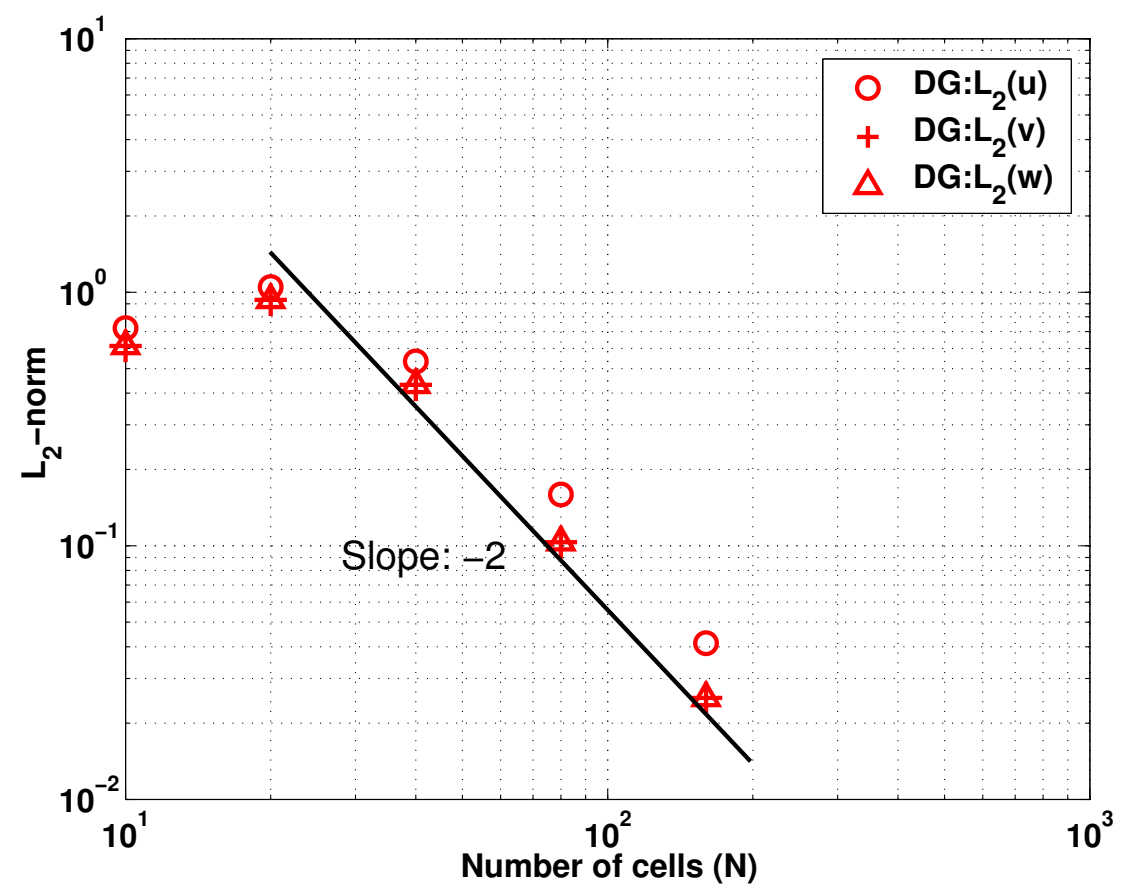

Figure 4. 2D GHHE Grid convergence study in the frozen limit for $r=s=0$ and $\epsilon=10^{3}$. 
that there is a term of higher-order in $\epsilon$ that is independent of $h$ that dominates the error for $h$ sufficiently small. Perhaps we have incorrectly dropped this term in our analysis, or it exists at an order higher than we have considered. We are still trying to understand the exact behavior but are satisfied that this result demonstrates the difficulty one may have in two or more dimensions.

\section{Discussion}

We have verified that, for both schemes in a single dimension, when $r \neq 0$, each has a mesh size threshold restriction $h \sim \epsilon^{1 / 3}|k|^{-2 / 3}$ above which numerical dissipation dominates and below which physical dissipation dominates. In each case, this result is consistent with a direct discretization of the advection-diffusion limit (4). It is this behavior that sometimes is identified in the literature as an obstacle to uniformly accurate schemes or asymptotic preserving schemes, depending on how the terms are defined.

For the case where $r \neq 0$, Lowrie and Morel, ${ }^{15}$ choose a diffusive scaling for their analysis and consider a different limit. There, a scaling more analogous to the incompressible limit $(r \ll 1)$ was used, where one can interpret the ratio of equilibrium to frozen wave speeds $r$ as a Mach number. ${ }^{\mathrm{b}}$ It was assumed further that $r=O(\epsilon)$ and that the Peclet number, $P e=r /\left(\epsilon\left(1-r^{2}\right)\right)$, was fixed. In this scaling, the inverse of the Peclet number is the coefficient of the diffusion term, and the frozen wave speeds scale like $1 / \epsilon$.

Lowrie and Morel defined their asymptotic preserving property this way: for $\epsilon \ll 1$, "the accuracy of the method should only depend on the resolution of scales related to $P e$, and not necessarily require resolution of scales corresponding to either $r$ or $\epsilon . "$ [15, p. 414]. That is, they seek to resolve the diffusion scale length, but not relaxation scale length (e.g., the mean-free path). In this sense, they demonstrated that the DG scheme was asymptotic preserving of the diffusive limit, while the HR scheme was not.

While this is consistent with the behavior we see for the DG method when $r=0$, it must be interpreted properly when $r=O(1)$ relative to $\epsilon$. If $r$ is fixed, $P e$ must go like $1 / \epsilon$, and one gets back the scaling used here. Rescaling Lowrie and Morel's truncation error analysis to our scaling, we recover our analytic results that lead to the convergence thresholds (37) and (41). Thus, in our scaling, both schemes fail to compute accurately the advection-diffusion limit if $h$ is completely independent of $\epsilon$ and $r$ is not small. This fact was identified by Lowrie and Morel, but interpreted as a reasonable restriction no worse than that imposed by direct discretization of the asymptotic advection-diffusion equation.

It seems reasonable to accept that, since the thresholds are no worse than the behavior one obtains by directly discretizing the advection-diffusion equation, that this is the best that can be expected. This has shown not to be the case by Jin and Levermore ${ }^{6}$ who devised a scheme for $r \neq 0$ that controlled this

\footnotetext{
${ }^{\mathrm{b}}$ This analogy is inexact and only suits our purposes for compressible gas dynamics. For other applications like radiation hydrodynamics, $r$ may be interpreted as the ratio of the hydrodynamic flow speed to the vacuum speed of light. The hydrodynamics in this case may still be compressible even for $r \ll 1$.
}

21 of 26

American Institute of Aeronautics and Astronautics Paper 2005-4989 


\begin{tabular}{cccccc}
\hline Method & $N_{x} \times N_{y}$ & $L_{2}(u)$ & Rate & $L_{2}(v)$ & Rate \\
\hline \multirow{2}{*}{ DG } & $5 \times 5$ & $5.78 \mathrm{e}-03$ & - & $2.34 \mathrm{e}-06$ & - \\
& $10 \times 10$ & $3.82 \mathrm{e}-04$ & 3.92 & $6.43 \mathrm{e}-07$ & 1.86 \\
& $20 \times 20$ & $4.52 \mathrm{e}-05$ & 3.08 & $1.65 \mathrm{e}-07$ & 1.97 \\
& $40 \times 40$ & $3.97 \mathrm{e}-05$ & 0.19 & $4.15 \mathrm{e}-08$ & 1.99 \\
& $80 \times 80$ & $3.94 \mathrm{e}-05$ & 0.01 & $1.06 \mathrm{e}-08$ & 1.96 \\
& $160 \times 160$ & $3.87 \mathrm{e}-05$ & 0.03 & $3.55 \mathrm{e}-09$ & 1.58 \\
\hline
\end{tabular}

Table 5. $L_{2}$-norm and rate of convergence for $r=s=0$ in the near-equilibrium limit $\left(\epsilon=10^{-5}\right)$ for the $2 \mathrm{D}$ GHHE system.

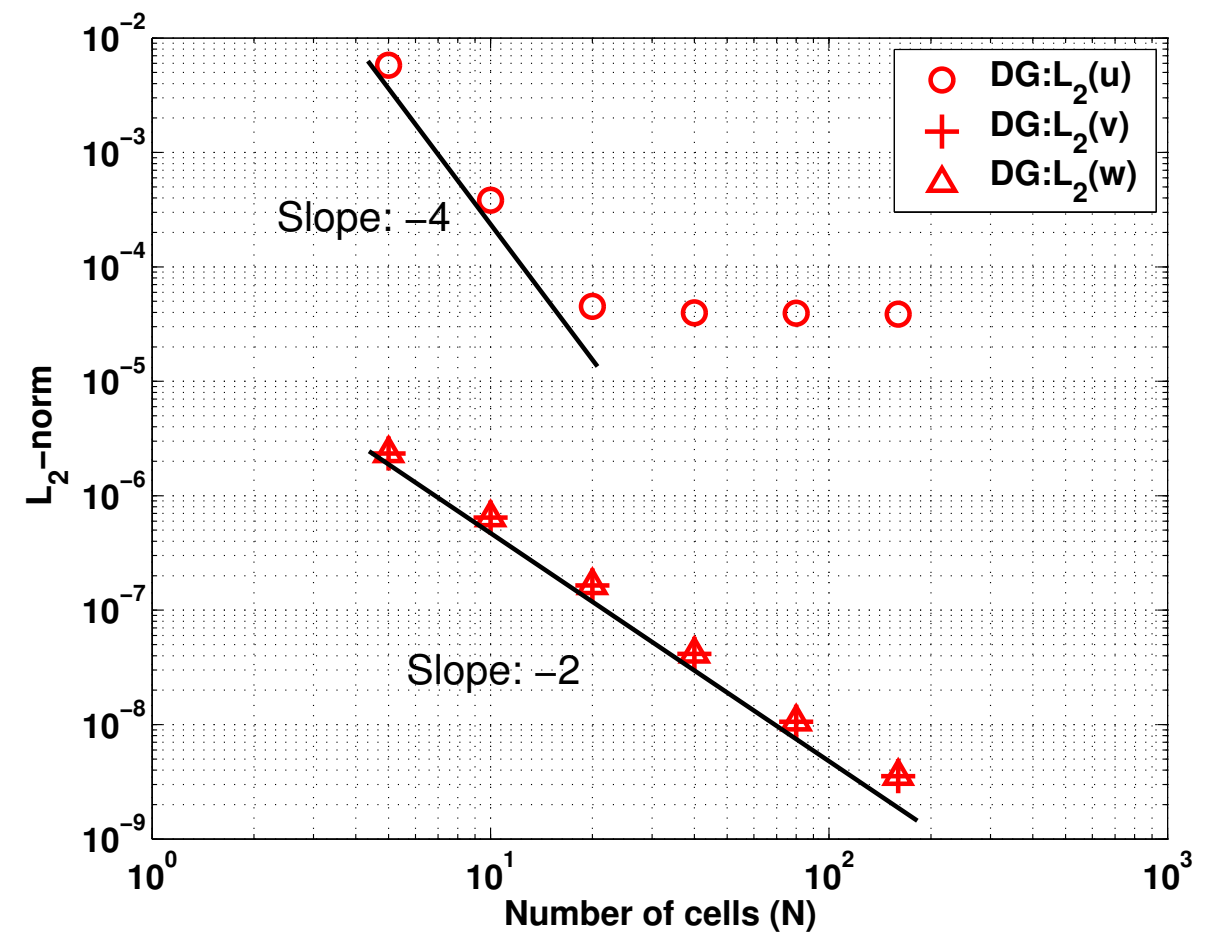

Figure 5. 2D GHHE grid convergence study in the near-equilibrium limit for $r=s=0$ and $\epsilon=10^{-5}$. 
dominant dissipation term by averaging the frozen and equilibrium fluxes. The averaging weight provided a free parameter that was used to make the numerical dissipation term scale with $\epsilon$. This suggests that the straightforward discretization fails to capture enough of the asymptotic coupling between the flux and source terms: Jin and Levermore force the flux discretization to include the asymptotic effects of relaxation on the flux. Furthermore, the DG scheme has less numerical dissipation than the HR scheme in the asymptotic limit, and the DG scheme includes more coupling between the two operators by directly evolving the slopes.

The most disconcerting result found in the simulations in 1D and 2D for the DG scheme for $r=0$ is that the convergence in $h$ of the numerical error slows or stops for $h$ sufficiently small. For a physically relevant compressible flow, $\epsilon$ will not be arbitrarily small to guarantee that successive mesh refinement will not lead to decreased order of convergence. We do not know yet the exact cause of this behavior.

\section{Summary and Future Work}

The Discontinuous Galerkin method has been applied to several model problems for hyperbolic systems with stiff relaxation in one and two spatial dimensions. The DG scheme, which has been shown previously to be asymptotic preserving of the diffusive limit, has been compared to the high-resolution Godunov scheme to suggest that the means by which the DG method preserves that asymptotic limit is the simultaneous advancement of the solution slopes and the solution averages. The asymptotic form of the DG flux function, when applied to a one-dimensional, asymmetric model problem has been shown to be equivalent to the first HLL flux function using the frozen wave speeds. Analysis for the advection-dominated advection-diffusion limit indicates that both the DG method and the HR method posses convergence thresholds that delay the onset of the second-order convergence. The numerical dissipation driving this feature is smaller for the DG method than for the HR method, and this must be a result of the direct evolution of solution slopes in the DG scheme. Grid convergence studies on simple linear model problems verify the analytic results that predict the mesh size $h$ relative to $\epsilon$ necessary to obtain second-order convergence.

For $r=0$, the DG scheme in one dimension converges with second-order accuracy with no apparent restriction on the grid size $h$ relative to the relaxation parameter $\epsilon$. In two dimensions, the convergence behavior for $r=s=0$ of the DG discretization is much more complicated; at first it appears to converge rapidly, but the convergence behavior twice transitions until the convergence apparently stalls.

There are many directions in which to proceed. We still must clarify the role of high-order terms in $\epsilon$ in the convergence behavior in both 1D and 2D. In addition, similar tests and analysis of the scheme of Jin and Levermore ${ }^{6}$ would be informative. Investigation of temporal effects and integrators must also be done.

Ultimately, two-dimensional problems must really be considered to demonstrate the proof of principle of using first-order systems as models for compressible, viscous flow. Flow transverse to the spreading shear 
layer generated by viscous or relaxation effects is the physically interesting problem, and one must consider at least two-dimension problems to see such phenomena.

\section{Acknowledgments}

The authors would like to thank R. Lowrie for his helpful discussions.

\section{A. 2D GHHE Dispersion Analysis}

Following the same procedure of dispersion analysis for the 1D model system, ${ }^{14}$ we assume a harmonic

$$
\mathbf{u}(x, y, t)=\mathcal{R}\left\{\mathbf{v}(t) \exp \left(-i\left[k_{x} x+k_{y} y\right]\right)\right\}
$$

where $\left(k_{x}, k_{y}\right)$ are wave numbers in $(x, y)$. Inserting this into (7) leads to an ordinary differential equation in terms of $\mathbf{v}(t)$,

$$
\frac{d \mathbf{v}(t)}{d t}=\left[i\left(k_{x} \mathbf{A}+k_{y} \mathbf{B}\right)+\mathbf{Q}\right] \mathbf{v}(t)
$$

The solution of this is

$$
\mathbf{v}(t)=\exp \left[i t\left(k_{x} \mathbf{A}+k_{y} \mathbf{B}-i \mathbf{Q}\right)\right] \hat{\mathbf{u}}_{0},
$$

where $\hat{\mathbf{u}}_{0} \in \mathbb{C}^{3}$ is a constant vector. The characteristic polynomial of the exponent is

$$
\begin{aligned}
\operatorname{det}\left[k_{x} \mathbf{A}+k_{y} \mathbf{B}-i \mathbf{Q}-\omega \mathbf{I}\right] & =0 \\
& =(i-\omega)\left(\omega^{2}-|k|^{2}-i\left[\omega+\left(r k_{x}+s k_{y}\right)\right]\right),
\end{aligned}
$$

where $|k|=\sqrt{k_{x}^{2}+k_{y}^{2}}$, and solutions are

$$
\begin{aligned}
\omega_{1} & =i \\
\omega_{2,3} & =\frac{i}{2}\left(1 \pm \sqrt{1+4\left[i\left(r k_{x}+s k_{y}\right)-|k|^{2}\right]}\right) .
\end{aligned}
$$

So long as $r k_{x}+s k_{y} \neq 0$ and $|k|^{2} \neq 1 / 4$, all three eigenvalues are distinct. In such a case, this matrix is diagonalizable, with right $\mathbf{R}$ and left $\mathbf{L}$ eigenvector matrices such that

$$
k_{x} \mathbf{A}+k_{y} \mathbf{B}-i \mathbf{Q}=\mathbf{R} \boldsymbol{\Omega} \mathbf{L}
$$


where $\boldsymbol{\Omega}=\operatorname{diag}\left(w_{1}, w_{2}, w_{3}\right)$, and for $\Delta k=\left(k_{x}-k_{y}\right)-i(r-s), \mathbf{L}=\mathbf{R}^{-1}$,

$$
\begin{gathered}
\mathbf{R}=\left(\begin{array}{ccc}
0 & k_{x}+k_{y} & k_{x}+k_{y} \\
k_{x} & \omega_{2}-\frac{k_{y}}{\omega_{3}} \Delta k & \omega_{3}-\frac{k_{y}}{\omega_{2}} \Delta k \\
-k_{y} & \omega_{2}+\frac{k_{x}}{\omega_{3}} \Delta k & \omega_{3}+\frac{k_{x}}{\omega_{2}} \Delta k
\end{array}\right), \\
\mathbf{L}=\frac{1}{|k|^{2}}\left(\begin{array}{ccc}
0 & \left(1+\frac{k_{x} \Delta k}{\omega_{2} \omega_{3}}\right) & -\left(1-\frac{k_{y} \Delta k}{\omega_{2} \omega_{3}}\right) \\
-\frac{\omega_{3}}{\omega_{2}-\omega_{3}} & \frac{k_{x}}{\omega_{2}-\omega_{3}} & \frac{k_{y}}{\omega_{2}-\omega_{3}} \\
\frac{\omega_{2}}{\omega_{2}-\omega_{3}} & -\frac{k_{x}}{\omega_{2}-\omega_{3}} & -\frac{k_{y}}{\omega_{2}-\omega_{3}}
\end{array}\right) .
\end{gathered}
$$

The solution of ODE $\mathbf{v}(t)$ can be written as

$$
\mathbf{v}(t)=\exp [i t(\mathbf{R} \boldsymbol{\Omega} \mathbf{L})] \mathbf{u}_{0}=\mathbf{R} \exp (i t \boldsymbol{\Omega}) \mathbf{L} \mathbf{u}_{0} .
$$

Inserting this into (57) gives the general solution at time $t$ :

$$
\mathbf{u}(x, y, t)=\mathcal{R}\left\{\mathbf{R} \exp (i t \boldsymbol{\Omega}) \mathbf{L} \mathbf{u}_{0} \exp \left(-i\left[k_{x} x+k_{y} y\right]\right)\right\}
$$

with the initial condition $\mathbf{u}_{0}$, which we define by $(56)$.

\section{References}

${ }^{1}$ Coirier, W. J. and Powell, K. G., "An Accuracy Assessment of Cartesian-Mesh Approaches for the Euler Equations," Journal of Computational Physics, Vol. 117, No. 1, pp. 121-131, 1995.

${ }^{2}$ Liu, T.-P., "Hyperbolic Conservation Laws With Relaxation," Communications in Mathematical Physics, Vol. 108, No. 1, pp. 153-175, 1987.

${ }^{3}$ Chen, G.-Q., Levermore, C. D., and Liu, T.-P., "Hyperbolic Conservation Laws With Stiff Relaxation Terms and Entropy," Communications on Pure and Applied Mathematics, Vol. 47, No. 6, pp. 787-830, 1994.

${ }^{4}$ Larsen, E. W., Morel, J. E., and Miller, Jr., W. F., "Asymptotic Solutions of Numerical Transport Problems in Optically Thick, Diffusive Regimes," Journal of Computational Physics, Vol. 69, No. 2, pp. 283-324, 1987.

${ }^{5}$ Larsen, E. W., and Morel, J. E., "Asymptotic Solutions of Numerical Transport Problems in Optically Thick, Diffusive Regimes II," Journal of Computational Physics, Vol. 83, No. 1, pp. 212-236, 1989.

${ }^{6}$ Jin, S., and Levermore, C. D., "Numerical Schemes for Hyperbolic Conservation Laws With Stiff Relaxation Terms," Journal of Computational Physics, Vol. 126, No. 2, pp.449-467, 1996.

${ }^{7}$ Arora, M., Explicit Characteristic-Based High-Resolution Algorithms for Hyperbolic Conservation Laws with Stiff Source Terms, Ph.D. Thesis, University of Michigan, Ann Arbor, Michigan, 1996.

${ }^{8}$ Pember, R. B., "Numerical Methods for Hyperbolic Conservation Laws With Stiff Relaxation: II. Higher-Order Godunov Methods," SIAM Journal on Scientific Computing, Vol. 14, No. 4, pp. 824-859, 1993.

${ }^{9}$ Lowrie, R. B. and Morel, J. E., "Discontinuous Galerkin for Stiff Hyperbolic Systems," AIAA paper 99-3307, 1999. 
${ }^{10}$ Caflisch, R. E., Jin, S., and Russo, G., "Uniformly Accurate Schemes for Hyperbolic Systems With Relaxation, SIAM Journal on Numerical Analysis, Vol. 34, No. 1, pp. 246-281, 1997.

${ }^{11}$ Pareschi, L., "Characteristic-Based Numerical Schemes For Hyperbolic Systems With Nonlinear Relaxation," Proceedings of the Ninth International Conference on Waves and Stability in Continuous Media, (Bari, 1997), Rendiconti del Circolo Matematico di Palermo (2) Suppl. 57, 1997, pp. 375-380.

${ }^{12}$ Bereux, F. and Sainsaulieu, L., "A Roe-type Riemann Solver for Hyperbolic Systems With Relaxation Based on TimeDependent Wave Decomposition," Numerische Mathematik, Vol. 77, No. 2, pp. 143-185, 1997.

${ }^{13}$ Liotta, S. F., Romano, V., and Russo, G., "Central Schemes for Balance Laws of Relaxation Type," SIAM Journal on Numerical Analysis, Vol. 38, No. 4, pp. 1337-1356, 2000.

${ }^{14}$ Hittinger, J. A., Foundations for the Generalization of the Godunov Method to Hyperbolic Systems with Stiff Relaxation Source Terms, Ph.D. Thesis, Univeristy of Michigan, Ann Arbor, Michigan, 2000.

${ }^{15}$ Lowrie, R. B. and Morel, J. E., "Methods for Hyperbolic Systems With Stiff Relaxation," International Journal for Numerical Methods in Fluids, Vol. 40, pp. 413-423, 2002.

${ }^{16}$ Naldi, G., and Pareschi, L., "Numerical Schemes for Hyperbolic Systems of Conservation Laws With Stiff Diffusive Relaxation," SIAM Journal on Numerical Analysis, Vol. 37, No. 4, pp. 1246-1270, 2000.

${ }^{17}$ Van Leer, B., Nomura, S., "Discontinuous Galerkin for Diffusion," AIAA-2005-5108, 17th AIAA Computational Fluid Dynamics Conference, June 2005.

${ }^{18}$ Hittinger, J. A. F. and Roe, P. L., "Asymptotic Analysis of the Riemann Problem for Constant Coefficient Hyperbolic Systems with Relaxation," Zeitschrift für Angewandte Mathematik und Mechanik, Vol. 84, No. 7, 2004, pp. $452-471$.

${ }^{19}$ Van Leer, B. "Stabilization of Difference Schemes for Hyperbolic Systems of Conservation Laws by Artificial Diffusion," Journal of Computational Physics, Vol. 3, pp. 473, 1969.

${ }^{20}$ Harten, A., Lax, P., and Van Leer, B., "On Upstream Differencing and Godunov-Type Schemes for Hyperbolic Conservation Laws," SIAM Review, Vol. 25, No. 1, pp. 35-61, 1983.

${ }^{21}$ Van Leer, B., "Towards the Ultimate Conservative Difference Scheme. IV. A New Approach to Numerical Convection," Journal of Computational Physics, Vol. 23, pp. 276-299, 1977. 